\title{
DETERMINATION DE LA QUALITÉ DU PAIN DE SUCRE A PARTIR DES CONCENTRATIONS DES SUCRES REDUCTEURS
}

\author{
M. Amrani \\ Departement de genie chimique, \\ Faculte des Sciences \& Techniques, BP 416, Tanger, Maroc
}

\begin{abstract}
RÉSUMÉ: Ce travail fait référence à un procédé automatisé pour l'élaboration du pain de sucre à partir du jus de canne à sucre. Les caractéristiques du produit traditionnel sont maintenues tout en veillant à conserver ses qualités intrinsèques qui en font un aliment naturel, peu altéré par ce procédé technique. Un procédé semi-continu a été développé pour élaborer le pain de sucre. Le procédé se subdivise en 3 étapes principales : l'extraction du jus de canne, l'épuration et la concentration du jus, enfin le moulage du pain de sucre. La dureté et la texture du produit fini sont les paramètres majeurs qui conditionnent la qualité du produit. Les fluctuations des concentrations en sucres réducteurs directs SRD influent significativement sur les paramètres dureté et texture. Ces derniers demeurent les indicateurs majeurs de critère de qualité du pain de sucre. Cette étude est un descriptif détaillé du procédé d'élaboration du pain de sucre dont la qualité dépend étroitement de la concentration en SRD tout au long de la chaîne de fabrication. Les corrélations entre les concentrations SRD et les paramètres qualité y sont également développés.
\end{abstract}

Mots clés: pain de sucre, process, qualité, sucre réducteurs, Maroc

\begin{abstract}
This work refers to an automated process for the development of the sugar loaf starting from the cane juice. The characteristics of the traditional product are maintained while taking care to preserve its intrinsic qualities which make of it a natural food, little modifed by this technical process. A semi-continuous process was developed to work out the sugar loaf. It is subdivided in 3 principal stages: the extraction of the cane juice, the purification and concentration of the juice, and finally the moulding of the sugar loaf. The hardness and the texture of the finished product are the major parameters which condition the quality of the sugar loaf. Fluctuations of concentrations of direct reducing sugars SRD have significative influence on the parameters hardness and texture. The latter remain the major indicators of quality standard of the sugar loaf. This study is a detailed description of the process of development of the sugar loaf whose quality depends closely on the concentration in SRD throughout the production line. The correlations between concentrations $S R D$ and the parameters quality are also developed there.
\end{abstract}

Key words: sugar loaf, process, quality, reducing sugar, Morocco

\section{INTRODUCTION}

Le pain de sucre est un produit élaboré à partir de la canne à sucre ou de la betterave (saccharum officinarum) qui présente une consistance solide moulée selon diverses formes. Dans d'autres pays comme la Colombie, connu sous le nom de « panela » ou en Inde « jaggery ou Gur», le pain de sucre est fabriqué à partir du jus de canne non raffiné (Martínez et al., 2004).
Au Maroc, le pain de sucre est ancré dans les habitudes de consommation des marocains. Il est utilisé essentiellement dans la préparation du thé à la menthe. Pendant longtemps, cette forme de présentation s'est confondue avec le sucre. Actuellement la consommation moyenne en sucre est de $32 \mathrm{~kg}$ par habitant dont $6 \mathrm{~kg}$ de pain de sucre par année (Cosumar, 2006). L'intérêt industriel et économique de cet aliment à l'échelle nationale est à l'origine de nombreuses études techniques et de recherche ciblant l'optimisation de ses qualités à la fois gustatives 
et texturales par analyse de ses paramètres physicochimiques. La dureté du pain de sucre est un des paramètres majeurs dans le processus d'élaboration de celui-ci. En effet cette dureté est directement liée aux concentrations en sucres réducteurs directs et totaux présents, respectivement SRD et SRT, conditionnant ainsi les critères de la qualité du pain de sucre (Velásquez et al., 2004). La présente étude est une analyse descriptive des sucres réducteurs directs et totaux dans la chaîne cinématique du processus de fabrication du pain de sucre à base de canne à sucre. Cette analyse descriptive singulière est fondamentalement basée sur l'étude de la texture du pain de sucre, laquelle est directement liée à la concentration en sucres réducteurs directs SRD.

\section{MÉTHODOLOGIE}

La détermination des Sucres Réducteurs Directs et Totaux (SRD \& SRT) a été effectuée grâce à la technique de Lane et Eyron du manuel «méthodes ICUMSA pour l'analyse du sucre » (Whalley, 1971). La concentration des SRD et SRT est le principal paramètre à mesurer avant et durant tout le processus: cette concentration est le paramètre indicateur de la qualité du pain de sucre. Ceci se reflète sur la dureté et la texture du produit fini. Les SRD sont les sucres contenant les carbones avec des groupes aldéhydes libres dans le cas du glucose et les groupes cétoniques dans le cas du fructose. La rupture de la liaison glucosique de la saccharose (liaison $\beta(1,2)$ ) produit les monomères glucose et fructose. Les SRT sont le résultat d'une hydrolyse totale effectuée sur les échantillons après acidification et chauffage (Whalley, 1971). La dureté du pain de sucre a été mesurée avec un texturomètre. Elle correspond à la résistance de celui-ci à une force externe exprimée en Newton.

\section{Processus de fabrication du pain de sucre}

L'élaboration et la production du pain de sucre est la synthèse de 3 étapes bien différenciées:

- Une première étape correspond à la préparation de la canne à sucre ou de la betterave et l'extraction du jus.

- La deuxième étape consiste en une épuration et une concentration de ce jus.

- $\quad$ La dernière en est le moulage du pain de sucre.

\section{Préparation de la canne à Sucre et extraction du jus}

La qualité du sucre produit dépend non seulement de la qualité de la betterave ou de la canne à sucre, mais aussi de la qualité du stockage de ces matières premières après la récolte.

Grâce à une succession d'opérations unitaires, on peut isoler le sucre (saccharose) des autres constituants de la betterave ou de la canne à sucre. En dehors des techniques de récolte (arrachage pour la betterave et coupe pour la canne à sucre), seules les premières étapes du procédé distinguent la sucrerie de canne de la sucrerie de betterave.

Il n'existe aucune différence entre les pains de sucre provenant de ces deux origines. Notre étude portera sur les pains de sucre fabriqués à partir du jus de canne.

L'extraction du jus de base se fait selon un protocole standard décrit sur la figure 1.

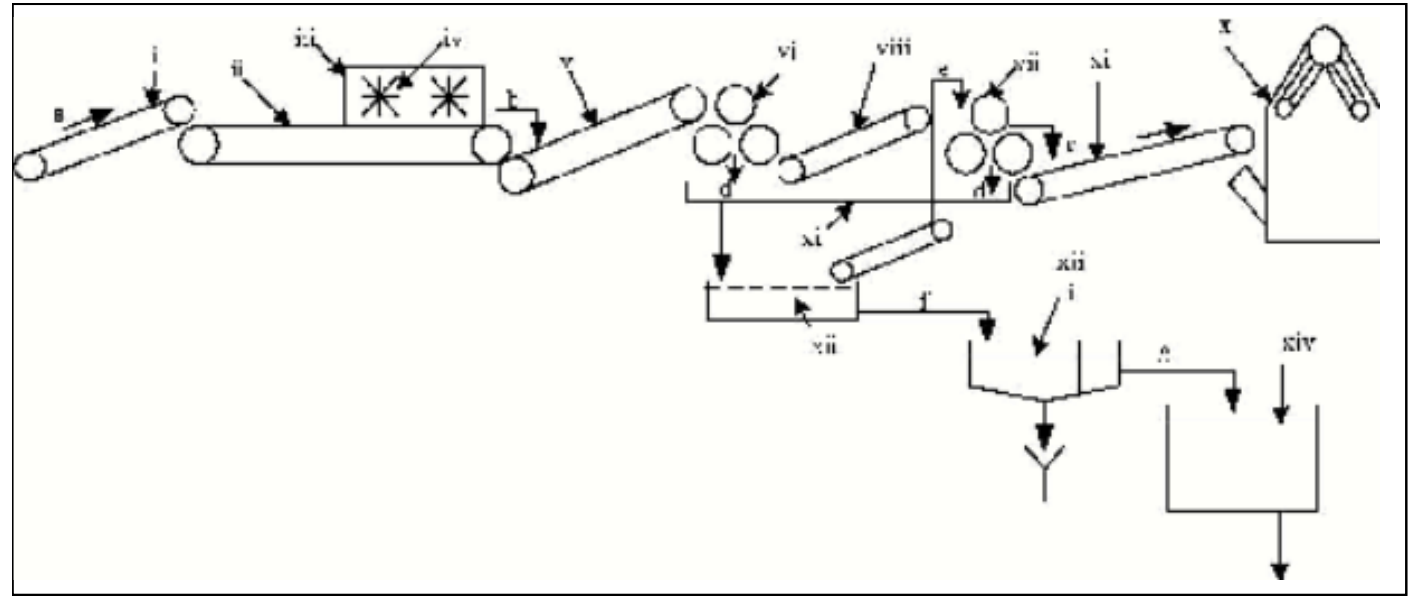

Figure 1 : schéma du processus de la préparation de la canne à sucre et extraction du jus (a) canne à sucre, (b) canne hachée et déchiquetée, (c) bagasse, (d) jus extrait, (e) particules de fine bagasse, (f) jus filtré, (g) jus préclarifié, (h) jus à épurer 
La canne à sucre est reçue puis préparée à travers des bandes transporteuses: elle est pesée, analysée pour déterminer sa richesse en saccharose, grâce à un échantillonnage effectué au sein des camions et engins de chargement et d'acheminement, ainsi que sa pureté. Elle est ensuite lavée afin d'éliminer la terre puis déchiquetée pour pouvoir en extraire le jus. Cette opération est effectuée dans des hacheurs («shredders »). Cette étape de broyage est déterminante car elle facilite considérablement le processus d'extraction.

Les morceaux de canne à sucre passent ensuite dans une série de trois moulins cylindriques montés en triangle et qui tournent lentement (4 à 6 tr/min).

Du premier moulin sort la première fraction du jus. La canne subit deux broyages dans chaque moulin. Le jus est récupéré au-dessous du groupe des moulins. Il est alors soit envoyé aux autres étapes de traitement (jus de première pression), soit réinjecté dans les moulins (jus mélangé). Le dernier moulin est interconnecté à une bande transportant la canne hachée presque sèche, appelée communément « bagasse », vers un silo de stockage afin d' être utilisée ultérieurement. Cette utilisation ultérieure de la «bagasse » prend diverses formes. Elle peut être utilisée comme combustible de chaudières pour la production d'électricité (Celeghini et al., 2000) et pour la production de l'éthanol. Enfin elle peut également servir en guise de complément dans l'alimentation animale (Pedrasa et al., 1996) particulièrement pour les ruminants. Actuellement, une application biotechnologique possible consiste en la production de xilitol et des solutions fermentables à base de bagasse hydrolysée.

Les moulins permettent d'extraire 92 à $96 \%$ de saccharose contenu dans la canne à sucre. Le jus, de couleur jaune verdâtre est riche en sucres réducteurs et en composés phénoliques. Pour éliminer une partie des impuretés, le jus est filtré par passage sur un épulpeur ou un tamis qui retient surtout les particules ligneuses, puis stocké dans un tank de décantation afin d'être épuré des précipités solides et des mousses accompagnant le jus.

\section{Epuration et concentration du jus de canne}

Les sucreries de canne ne réalisent pas d'épuration calcocarbonique à proprement parler mais plutôt une alcalinisation progressive du jus jusqu'à un $\mathrm{pH}$ donné.
Tout en étant agité, le jus est préchauffé jusqu'à une température de $90^{\circ} \mathrm{C}$. On ajoute 200 à $300 \mathrm{ppm}$ d'acide phosphorique à $75 \%$ puis on effectue un traitement à la chaux appelé communément « chaulage ». Cette opération permet d'ajuster le $\mathrm{pH}$ à des valeurs comprises entre 6.2 et 7.0 (Hugot, 1986)(Chen, 2000).

Le bac de formulation à son tour est relié au moyen de tuyauteries à un système de décanteurs(xvi) où sédimentent les substances indésirables. Ces décanteurs, interconnectés entre eux, évacuent le jus clarifié dans un réservoir récepteur (xvii) pour concentration.

A la fin de cette opération, la concentration en SRD du jus clarifié est déterminée pour évaluer l'augmentation de la concentration.

Le circuit d'évaporation consiste en deux évaporateurs (xviii et xix) qui permettent de concentrer le jus épuré jusqu'à l'obtention d'un sirop à une concentration voisinant la saturation. La vapeur générée dans le premier évaporateur permet de réchauffer le jus à l'entrée du second évaporateur comme le montre la figure 2 .

L'évaporation dans le premier évaporateur se fait sous pression constante de $500 \mathrm{~mm} \mathrm{Hg}$ à $75^{\circ} \mathrm{C}$. Le deuxième évaporateur fonctionne à $500 \mathrm{~mm} \mathrm{Hg}$ et à $55^{\circ} \mathrm{C}$.

Le jus, entrant dans les évaporateurs à une concentration variant de 18 à $20^{\circ} \mathrm{Bx}$, en ressort à des concentrations atteignant $75^{\circ} \mathrm{Bx}$.

La concentration en SRD est déterminée à la fin de cette opération afin de préserver la qualité du produit. Le jus concentré est placé dans un tank de stockage (xx) avant de passer dans un évaporateur à pression atmosphérique où la concentration du jus se fait à $120^{\circ} \mathrm{C}$, atteignant $92^{\circ} \mathrm{Bx}$.

\section{Moulage et emballage}

Le jus concentré est envoyé vers un tank récepteur (xxiii) muni d'un agitateur à vitesse variable tel que présenté sur la figure 3. Ce système a pour but de forcer la cristallisation du saccharose et de baisser la température jusqu'à $85^{\circ} \mathrm{C}$. Ce procédé permet de garantir une meilleure manipulation de la masse cuite et une réduction du temps de moulage et de cristallisation finale. 


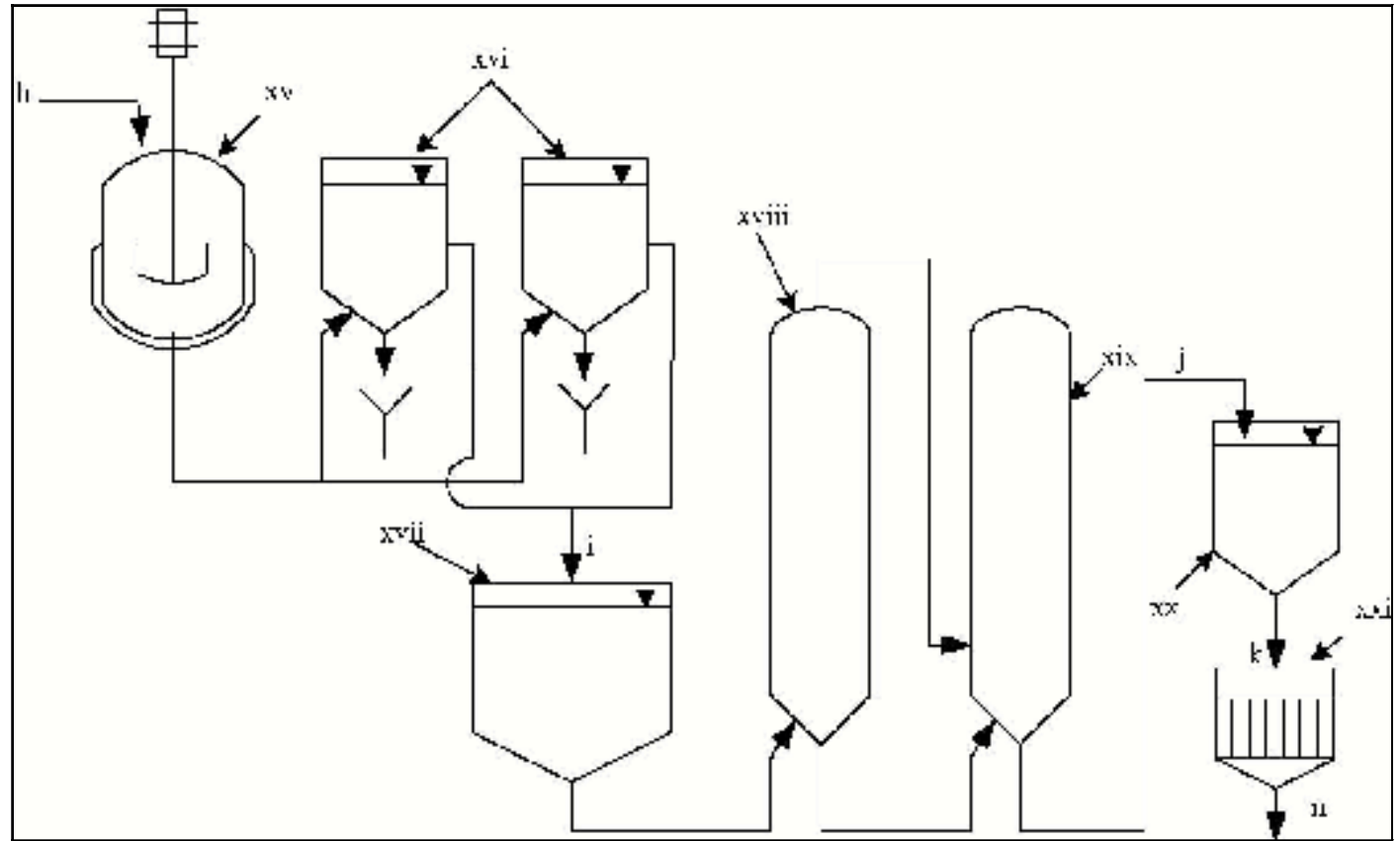

Figure 2: schéma d'épuration et de concentration.

(h) jus à épurer ; (i) jus épuré; ; (j) jus concentré de 65 à $74^{\circ} \mathrm{Bx}$; (k) jus concentré de $92^{\circ} \mathrm{Bx}$. $\square$ Le degré Brix $\left({ }^{\circ} \mathrm{Bx}\right)$ est le poids en grammes de matières sèches contenues dans 100 grammes d'une solution dans l'eau distillée

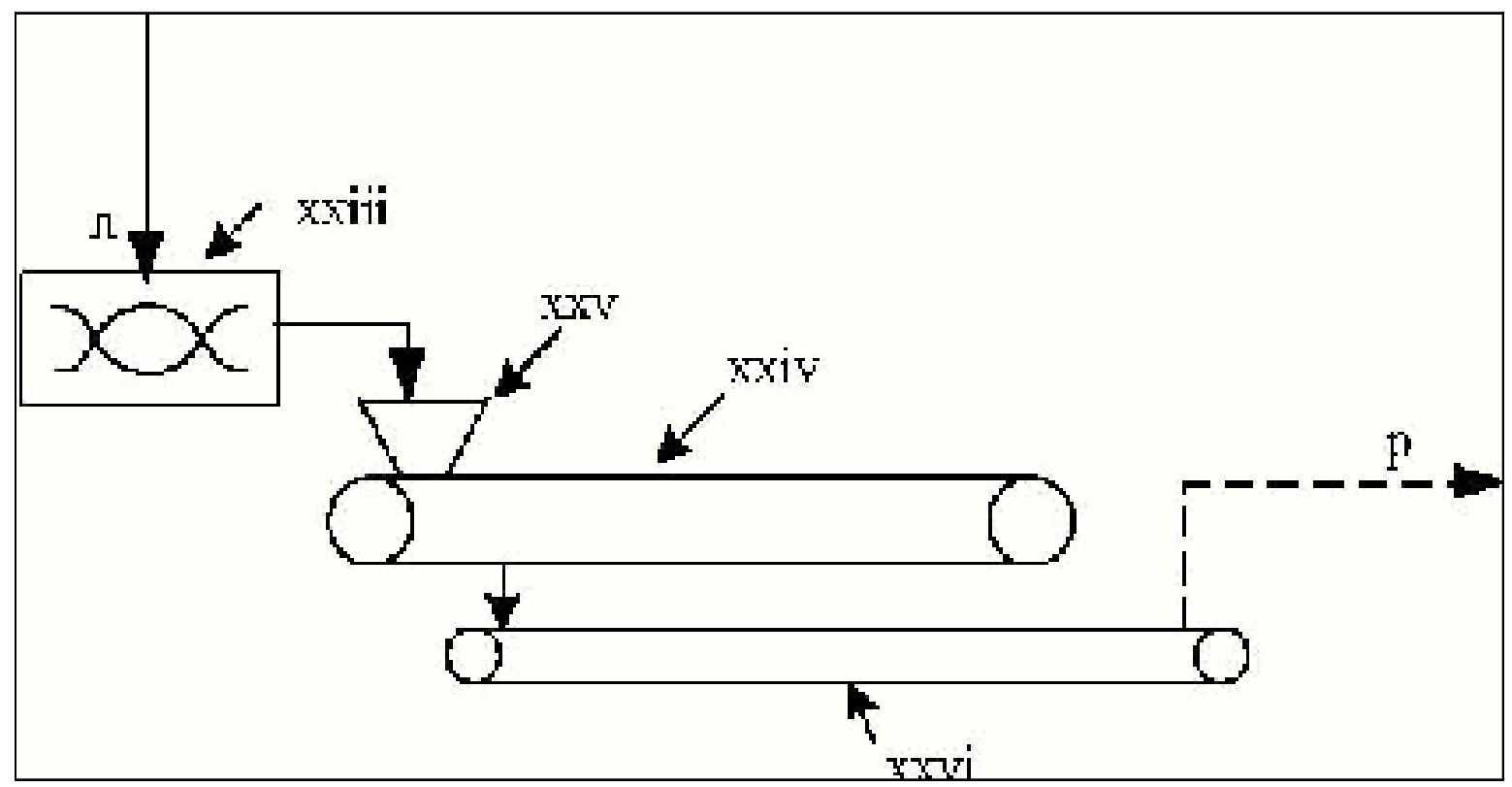

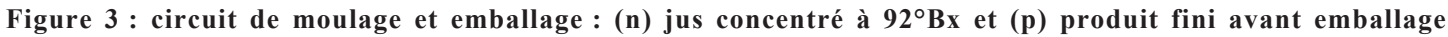


La masse cuite est ensuite coulée dans des formes métalliques transconiques anti adhésives (revêtement en Téflon) (xxiv) à travers une trémie (xxv) maintenue à $85^{\circ} \mathrm{C}$. Les moules de pain de sucre sont refroidis progressivement sur la bande transporteuse (xxvi). Le produit solide subit alors un turbinage, c'est à dire une séparation des phases liquide et solide par centrifugation des formes, un démoulage ou lochage puis un sciage ou sont découpées les rondelles des pains de sucre en surpoids. Les pains de sucre sont ensuite emballés dans du papier (Figure 4).

Traditionnellement, les pains de sucre se présentaient sous forme de blocs solides avec diverses formes et taille. Pour des facilités de manipulation et de moulage, la forme transconique a été largement adoptée. La qualité du pain de sucre fabriqué dépend de la concentration en SRD et de la dureté du produit final.
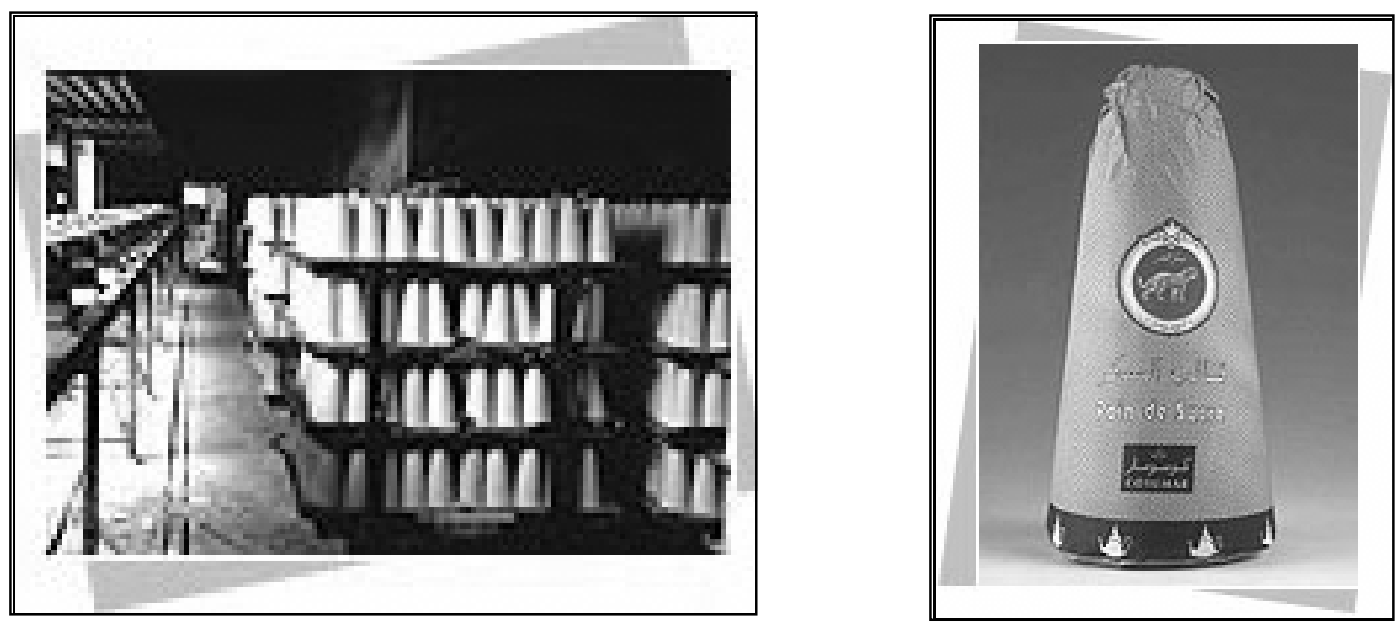

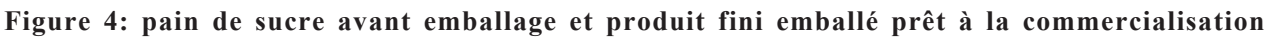

\section{RÉSULTATSETDISCUSSION}

Le processus d'épuration a été effectué suite à une alcalinisation progressive du jus en utilisant de la chaux et de la chaleur pour décanter le jus et précipiter les impuretés noires ou les « boues ». L'utilisation de la chaux permet de neutraliser les acides organiques contenus dans le jus de canne ( Hugot, 1986 ) (Chen, 2000). Le processus d'épuration permet aussi de séparer les mousses ou écumes riches en sels de calcium insolubles, l'albumine coagulée, les graisses et cire ainsi que les fines de bagasse («bagacillo )). Les températures lors de l'épuration peuvent atteindre $95^{\circ} \mathrm{C}$. Il existe d'autres méthodes innovatrices proposées pour l'épuration du jus de la canne à sucre comme celle consistant en un « chaulage » à froid qui induit une amélioration de la couleur du jus de canne, une baisse de la concentration en sucres invertis en dextrane et en oligosaccharides (Eggleston et al., 2002).

Lors de l'épuration du jus de canne, la concentration en sucres réducteurs directs varie très peu comme le montre les résultats comparatifs du jus de canne avant et après l'épuration (tableau 1).

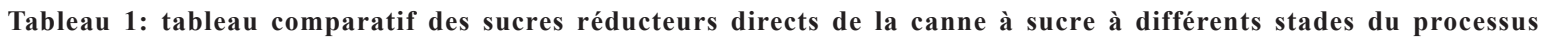

\begin{tabular}{|c|c|c|c|c|c|c|c|c|c|c|c|c|c|c|c|}
\hline \multicolumn{4}{|c|}{$\begin{array}{c}\text { jus de canne } \\
\text { avant épuration }\end{array}$} & \multicolumn{4}{|c|}{ jus de canne épuré } & \multicolumn{4}{|c|}{ jus de canne concentré } & \multicolumn{4}{|c|}{ pain de sucre fini } \\
\hline${ }^{\circ} \mathrm{Bx}$ & $\begin{array}{l}\text { SRD } \\
(\%)\end{array}$ & $\begin{array}{l}\text { SRT } \\
(\%)\end{array}$ & $\begin{array}{c}\text { SRD/SRT } \\
(\%)\end{array}$ & ${ }^{\circ} \mathrm{Bx}$ & $\begin{array}{l}\text { SRD } \\
(\%)\end{array}$ & $\begin{array}{l}\text { SRT } \\
(\%)\end{array}$ & $\begin{array}{c}\text { SRD/SRT } \\
(\%)\end{array}$ & ${ }^{\circ} \mathrm{Bx}$ & $\begin{array}{l}\text { SRD } \\
(\%)\end{array}$ & $\begin{array}{l}\text { SRT } \\
(\%)\end{array}$ & $\begin{array}{c}\text { SRD/SRT } \\
(\%)\end{array}$ & ${ }^{\circ} \mathrm{Bx}$ & $\begin{array}{c}\text { SRD } \\
(\%)\end{array}$ & $\begin{array}{l}\text { SRT } \\
(\%)\end{array}$ & $\begin{array}{c}\text { SRD/SRT } \\
(\%)\end{array}$ \\
\hline 20 & 0.6 & 17.6 & 3 & 21 & 0.61 & 18.3 & 3 & 74 & 2.2 & 64.4 & 3 & 94 & 7.1 & 82.6 & 3 \\
\hline 19 & 0.6 & 15.5 & 4 & 21 & 0.58 & 16.6 & 3 & 73 & 2. & 57.8 & 4 & 92 & 6.8 & 73.5 & 3 \\
\hline 19 & 0.4 & 15.5 & 3 & 20 & 0.52 & 15 & 3 & 72 & 2.1 & 57 & 4 & 89 & 8.2 & 71.0 & 3 \\
\hline 21 & 4.3 & 17.9 & 24 & 21 & 4.4 & 17.9 & 25 & 74 & 16 & 63.1 & 25 & 95 & 27.3 & 80.9 & 3 \\
\hline 21 & 1.8 & 16.8 & 10 & 22 & 1.93 & 17.5 & 11 & 72 & 7.1 & 58.5 & 12 & 91 & 14.0 & 72.6 & 3 \\
\hline
\end{tabular}


Durant le processus de fabrication du pain de sucre, il est important de contrôler les concentrations en sucres réducteurs directes. La nécessité du suivi à différentes étapes du procédé afin d'éviter l'hydrolyse du saccharose contenue dans le jus de canne s'y impose naturellement.

Durant le processus de concentration du jus épuré, le saccharose peut subir une hydrolyse provoquée par la chaleur appliquée aux deux évaporateurs (à double effet). L'hydrolyse de la saccharose est en relation directe avec le rapport des sucres réducteurs directs et des sucres réducteurs totaux. (SRD/SRT). Ce rapport augmente au niveau des étapes du processus ou le mélange est chauffé, de façon considérable. L'augmentation de l'hydrolyse de la saccharose est due à la rupture des liaisons glucosiques, liaisons faibles car elles ne nécessitent que $26 \mathrm{kcal} / \mathrm{mol}$ pour rompre la liaison entre le glucose et le fructose ( Badui, 1995 ).

Lors du moulage du pain de sucre, le jus concentré est amené à des températures supérieures à $100^{\circ} \mathrm{C}$ : ceci provoque une hydrolyse encore plus importante que dans le jus de canne. Le rapport SRD/SRT augmente de 2.4 fois en moyenne dans les différentes configurations depuis l'obtention du jus épuré jusqu'au pain de sucre final.

Le rapport SRD/SRT dans le produit final a pour conséquence une consistance rigide du pain de sucre ; mais si cette relation dépasse $10 \%$, la consistance est alors équivalente à celle d'un fluide non newtonnien avec un profil de gel très visqueux, difficile à manipuler et à mouler.

Les SRD ont la particularité de ne pas être cristallisables, exerçant ainsi un effet inhibitoire sur la cristallisation du saccharose ( Badui, 1995 ). La concentration élevée des SRD dans le pain de sucre affecte principalement sa dureté, paramètre caractérisant la qualité du produit fini. Des essais de dureté du pain de sucre ont montré que pour certains échantillons, une concentration moyenne en SRD correspondait à une force appliquée de $159 \mathrm{~N}$. Lorsque la concentration en SRD augmente respectivement de 8.2\%, $14 \%$ et $19.4 \%$, cette force correspondant respectivement à des intensités de 92,41 et $35 \mathrm{~N}$ mesurées par un texturomètre.

Cette information nous permet de supposer que plus la concentration en SRD est élevée, plus le pain de sucre est fragile et mou : une sollicitation faible est suffisante pour le déformer ou le casser.

Pendant le processus d'élaboration du pain de sucre, un contrôle continu est nécessaire lors de l'épuration et de la concentration du jus de canne. La qualité de la canne à sucre est un facteur primordial dans la fabrication du pain de sucre. La meilleure canne à sucre se décline conjointement sous deux formes : celle atteignant une certaine maturité ( à savoir 11 à 12 mois) et celle pour laquelle la concentration en saccharose est maximale. Ainsi la canne à sucre doit être traitée immédiatement après la coupe afin d'éviter sa détérioration mécanique, bactériologique ou encore l'inversion du saccharose et de la fonction dextrane par les microorganismes tels les leuconostoc mesenteroides ( Chen., 2000 ) (ArguelloMorales et al., 2005) . L'intérêt de traiter une canne fraîchement coupée et de bonne qualité en est ainsi fortement recommandé.

\section{CONCLUSION}

La production du pain de sucre a été adoptée au Maroc depuis longtemps et de manière artisanale avant d'être de nos jours entièrement industrialisée. La qualité du pain de sucre dépend principalement de la concentration en sucres réducteurs directs qui agissent directement sur la dureté du produit final, indépendamment de la couleur puisque la dureté du pain est meilleure si la concentration en SRD est faible. Lors des étapes d'extraction et d'épuration du jus de canne, la concentration en SRD ne subit pas de changements significatifs, contrairement à l'étape du moulage où la concentration en sucres réducteurs varie de manière plus importante à cause de l'élévation de la température. Cette élévation de température, provoque en conséquence l'hydrolyse de certaines molécules de saccharose. La chaleur fournie par élévation de température s'avère nécessaire pour atteindre la sursaturation de la concentration en saccharose permettant ainsi d'atteindre le seuil de cristallisation.

\section{RÉFÉRENCES}

Arguello-Morales, M., Sanchez-Gonzales, M., Canedo, M., Quirasco, M., Farres, A., Lopez-Munguia, A., (2005), Proteolytic modification of Leuconostoc mesenteroides B-512F dextransucrase, Antonie Van Leewenhoek, 87(2):131-141.

Badui, J.D.S., (1995), Quimica de alimentos, 3 a edicion, 62-65, Edition Alambra Mexicana, Mexico.

Celeghini, E.A, Dos Santos Celeghini \& Lanças F.M, (2000), Experimental variables and their effects on the liquefaction of cellulose from sugar cane bagasse, Energy Sources, 23:93-99.

Chen., C.P., (2000), Manual del azúcar de caña, 32-37,4143, 163-190, Edicion Limusa, Mexico.

Cosumar, (2006), Rapports Internes, Compagnie Sucrière du Maroc, Cosumar, Groupe ONA, http:// www.consumar-ona.ma 
Eggleston, G., Monge, A., Ogier, E., (2002), Sugar factory performance of cold, intermediate and hot lime clarification process, J.Proces.Preservation, 26:433454.

Hugot, H., (1986), Handbook of cane sugar engineering, Third Edition, 399-416, Elsevier, Amsterdam.

Martínez, H., Acevedo, X., (2004). Caracteristicas y estructura de la cadena agroindustrial de la panela en Colombia. Documento de trabajo No. 12. Ministerio de Agricultura y Desarrollo Rural, Bogotá, Colombia.
Pedrasa, R.O., Zafra, L.M., Ramos Sanchez, L.B., (1996), Método de obtención de un alimento para el ganado a partir de la caña de azucar, Patent $\mathrm{N}^{\circ} \mathrm{CU} 22337 \mathrm{AI}$, Oficina Cubana de la Propriedad Industrial.

Velásquez, H. I., J. F. Chejne y S. A. F. Agudelo, (2004). Diagnóstico energético de los procesos productivos de la panela en Colombia. Revista Facultad Nacional de Agronomía, Vol. 57, No. 2. Medellín, Colombia. 